\title{
Solving Einstein's equations with dual coordinate frames
}

\author{
Mark A. Scheel, ${ }^{1}$ Harald P. Pfeiffer, ${ }^{1}$ Lee Lindblom, ${ }^{1}$ Lawrence E. Kidder, ${ }^{2}$ Oliver Rinne, ${ }^{1}$ and Saul A. Teukolsky ${ }^{2}$ \\ ${ }^{1}$ Theoretical Astrophysics 130-33, California Institute of Technology, Pasadena, California 91125, USA \\ ${ }^{2}$ Center for Radiophysics and Space Research, Cornell University, Ithaca, New York 14853, USA
}

(Received 13 July 2006; published 2 November 2006)

\begin{abstract}
A method is introduced for solving Einstein's equations using two distinct coordinate systems. The coordinate basis vectors associated with one system are used to project out components of the metric and other fields, in analogy with the way fields are projected onto an orthonormal tetrad basis. These field components are then determined as functions of a second independent coordinate system. The transformation to the second coordinate system can be thought of as a mapping from the original inertial coordinate system to the computational domain. This dual-coordinate method is used to perform stable numerical evolutions of a black-hole spacetime using the generalized harmonic form of Einstein's equations in coordinates that rotate with respect to the inertial frame at infinity; such evolutions are found to be generically unstable using a single rotating-coordinate frame. The dual-coordinate method is also used here to evolve binary black-hole spacetimes for several orbits. The great flexibility of this method allows comoving coordinates to be adjusted with a feedback control system that keeps the excision boundaries of the holes within their respective apparent horizons.
\end{abstract}

DOI: 10.1103/PhysRevD.74.104006

PACS numbers: 04.25.Dm, 02.60.Cb, 04.20.Cv, 04.70.Bw

\section{INTRODUCTION}

This paper introduces a new method of solving Einstein's equations using two distinct coordinate systems. Tensors like the metric are represented by their components in the coordinate basis of the first coordinate system. Einstein's equations are then used to determine these tensor components as functions of the second coordinate system. The mapping between the first and second coordinate systems is quite arbitrary and can be chosen dynamically. This freedom allows us to adapt the second coordinate system continuously, for example, to track the motion of the individual black holes in a binary. The first coordinate system plays much the same role as the orthonormal tetrad used in some formulations of the Einstein equations [1-3]. The transformation to the second coordinate system can be thought of as a mapping between the original "inertial" coordinates and the computational domain, and plays much the same role as the "grid velocity" sometimes used in numerical hydrodynamics [4]. The bulk of this paper gives a careful description of this new method as it applies to the generalized harmonic form of the Einstein equations [5], plus a set of numerical tests that demonstrate its usefulness.

The flexibility to choose a coordinate frame adapted to a particular physical problem is often used to simplify solutions of the Einstein equations. For example, solutions having some symmetry (e.g., time independence) are much simpler when expressed in coordinates that respect that symmetry. It has long been expected that binary blackhole spacetimes would most naturally be represented in a coordinate frame that corotates with the orbit of the holes; this frame should be advantageous for numerical simulations since it would make the fields nearly time independent during the inspiral. Unfortunately our attempts to model binary black-hole spacetimes in the conventional single-coordinate framework have all failed. These failures reveal several serious and interesting problems with the single-frame approach, and motivated us to develop the new methods described in this paper. Before we turn to our discussion of the new dual-frame approach, however, we believe it is useful to describe those problems with the standard single-frame methods.

The groundbreaking binary black-hole evolutions of Pretorius [6] use a single coordinate frame that asymptotically approaches the inertial frame at infinity. The singular interior regions of the holes are excised from the computational domain. In this approach the black holes move across the coordinate grid and the solution is time dependent on the orbital time scale. We have attempted to implement a similar scheme using our spectral evolution code, but encountered two significant problems: first, the blackhole "excision" boundaries must be changed from time to time to track the motions of the black-hole horizons. This requires finding a way of adding (and deleting) grid points to (and from) the computational domain in a way that keeps the distance between the horizon and the excision boundary more or less fixed, and then - the difficult part to find sufficiently smooth and stable ways to fill (e.g., by extrapolation) the new grid points with appropriate values for all the dynamical fields. This problem has been solved successfully for lower-order finite-difference numerical methods [6-10]. The difficulty for the spectral-method implementation seems to be the inability to produce suffi- 
ciently smooth and well-behaved extrapolations of the needed dynamical field quantities to points lying beyond the current computational domain. Our attempts to develop such techniques have not been successful.

There is also a second fundamental problem with the conventional moving-excision method: although the blackhole horizons move through the computational grid, the excision boundaries are fixed except when they are moved to update the grid. To understand why this can cause trouble, consider first an excision surface located just inside the horizon, but moving continuously along with the horizon. In this case, the excision boundary is a spacelike surface that lies in the future of the computational domain, so (for a causal hyperbolic representation of the Einstein equations) boundary conditions are not needed there, and the spacetime region inside can be excised. Now consider the conventional moving-excision method, where the boundaries are fixed in the coordinate grid during a time step, and therefore move relative to the horizons. At the trailing edge of the horizon, the excision surface is moving out of the black hole superluminally. Locally this surface is spacelike and lies in the future of the computational domain, so boundary conditions are not needed there. But at the leading edge, the excision surface is falling deeper into the black hole; if it falls quickly enough, some part of this surface may become timelike, so boundary conditions will be required at these points. If appropriate boundary conditions are not supplied, the evolution problem becomes ill-posed and the black-hole excision paradigm breaks down. We monitor the characteristic speeds at excision boundaries in our code, and have verified that this breakdown does occur in our simulations when a black hole moves too quickly through the coordinate grid. This problem can be ameliorated by moving the excision boundaries deeper into the black-hole interiors (where with an appropriate choice of gauge, light cones are "tipped" further toward the singularity); we presume this is how the successful finite-difference codes control this problem. But we were not successful in curing this problem in our spectral evolution code. Another successful approach to moving black holes through the computational grid is the movingpuncture method, cf. Refs. [11-16], which can be thought of as shrinking the internal excision boundaries to isolated points that lie between the grid points (and so are simply ignored). Unfortunately the dynamical fields are not smooth at the puncture points, so the moving-puncture approach may not be extendable to spectral methods.

Since we were unsuccessful in moving black holes through our spectral computational domain using the conventional method, we were led to pursue an approach using comoving coordinates where the black holes remain at rest. Unfortunately, the generalized harmonic evolution system [5] exhibits severe instabilities (in the sense that the constraints grow without bound) when initial data are evolved using coordinates that rotate with respect to the inertial

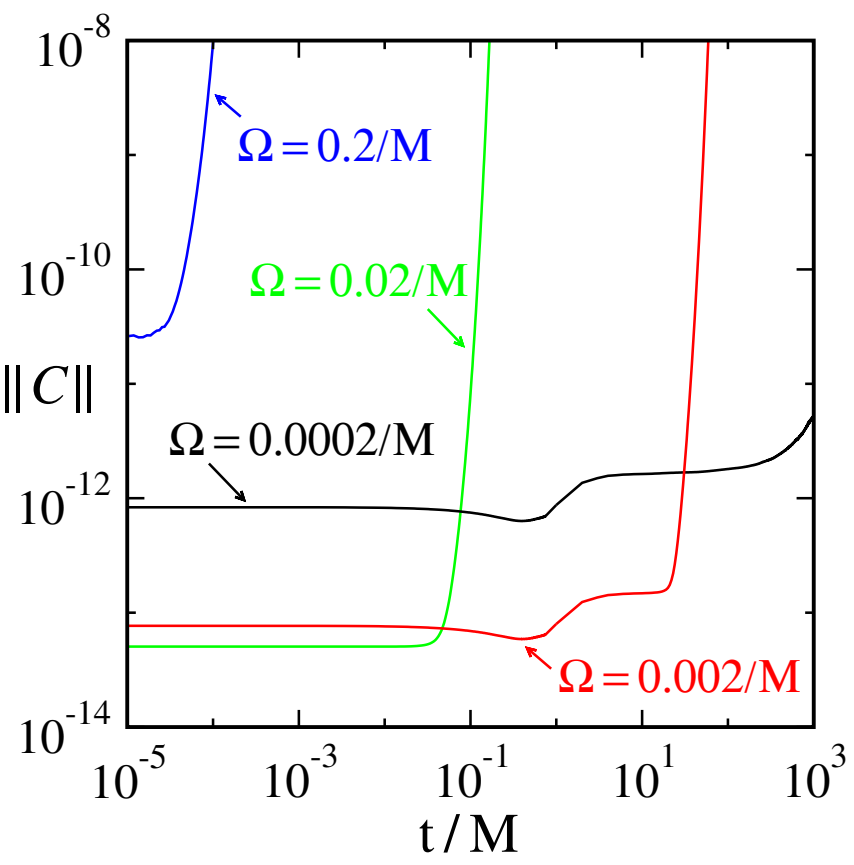

FIG. 1 (color online). Constraint violations in evolutions of a Schwarzschild black hole using the generalized harmonic system and coordinates that rotate with angular velocity $\Omega$.

frame at infinity. ${ }^{1}$ These instabilities occur even for simple time-independent cases like Minkowski or Schwarzschild spacetime. For example, Fig. 1 shows the constraint violations for evolutions of Schwarzschild in a frame rotating with angular velocity $\Omega$. These evolutions were performed on a computational domain that is a little larger than the size needed for binary black-hole evolutions, with outer radius $R_{\max }=1000 M$; see Sec. III for details. Binary black-hole evolutions must be performed stably and accurately for times of order $1000 \mathrm{M}$ to model all of the interesting inspiral and merger dynamics. However, the rotating frame evolution shown in Fig. 1 with $\Omega=0.2 / M$ (comparable to the maximum angular velocity achieved by a black-hole binary just before merger) is unstable in the unacceptably short time of about $10^{-4} \mathrm{M}$. We do not fully understand the instability in evolving asymptotically flat spacetimes in rotating coordinates using the generalized harmonic evolution system. We have reduced the severity of this problem with methods discussed in the Appendix,

\footnotetext{
${ }^{1}$ This instability appears to be a feature of the generalized harmonic system. No instability was seen in earlier evolutions of black holes in rotating frames using other forms of the Einstein system [17], and we do not find such an instability using our code with the Kidder-Scheel-Teukolsky (KST) evolution system [18]. In the generalized harmonic system the full 4-metric, including the lapse and shift, are evolved as dynamical fields, while in the KST system only the 3-metric is evolved. Since only the shift grows near spatial infinity in a rotating frame, the nondynamical treatment of the shift in the KST system may account for its rotating frame stability.
} 
but we were not able to solve completely the problem of evolving with a single corotating coordinate frame.

Another fundamental limitation on using rotating coordinates in asymptotically flat spacetimes is a numerical resolution issue: some components of the four-metric grow asymptotically like $\rho^{2} \Omega^{2}$ for large values of the cylindrical radial coordinate $\rho$ (to leading order), while other components approach $M / r$, for large values of the spherical radial coordinate $r$. On computational domains that extend to $r \approx$ $1000 M$ and angular velocities $\Omega \approx 0.2 / M$ (appropriate for binary black-hole spacetimes), these values differ by almost 8 orders of magnitude, so that the dynamical range needed to resolve the various field components with sufficient accuracy is difficult to achieve using double precision numerical methods. This difficulty goes away if one uses the inertial-frame components of the various fields [6].

The dual-frame approach proposed here corrects or circumvents all of the problems associated with the more traditional approaches that we know about. The remainder of this paper is organized as follows. The dual-coordinate frame method is presented more completely in Sec. II. This new method is applied in Sec. III to the case where the two coordinate frames rotate uniformly with respect to each other. We test this new rotating-coordinate method by evolving Schwarzschild initial data in coordinates that rotate with respect to infinity, finding that the evolutions of Fig. 1 become stable and convergent. A number of technical numerical issues associated with the dualcoordinate method (e.g., how to construct the appropriate spectral filters) are also discussed in Sec. III. Section IV further tests this new method by evolving a binary blackhole spacetime in uniformly rotating coordinates. This test fails when the fixed angular velocity of the rotating frame no longer tracks the motion of the black holes to sufficient accuracy. In Sec. V we present a more sophisticated application of the dual-coordinate method by constructing a flexible coordinate map whose parameters are adjusted through a feedback control system that keeps the blackhole horizons centered on the excision surfaces. Using these new horizon-tracking coordinates, we are able to evolve a binary black-hole spacetime in a stable and convergent manner for about 4.6 orbits. Our results are summarized, and a discussion of possible directions for further development are given in Sec. VI. Finally, we review in the Appendix our attempts to make rotating single-coordinate frame evolutions stable for the generalized harmonic evolution system.

\section{DUAL-COORDINATE FRAMES}

Consider a first-order representation of the Einstein evolution system, such as our formulation of the generalized harmonic system [5]. The evolution equations for the dynamical fields $u^{\bar{\alpha}}$ for such systems can be represented abstractly as

$$
\partial_{\bar{t}} u^{\bar{\alpha}}+A^{\bar{k} \bar{\alpha}} \bar{\beta}_{\bar{\beta}} u^{\bar{\beta}}=F^{\bar{\alpha}} \text {, }
$$

where $A^{\bar{k} \bar{\alpha}}{ }_{\bar{\beta}}$ and $F^{\bar{\alpha}}$ may depend on $u^{\bar{\alpha}}$ but not its derivatives. We use Greek indices $\bar{\alpha}, \bar{\beta}, \ldots$ to label the various dynamical fields, and Latin indices $\bar{l}, \bar{j}, \bar{k}, \ldots$ to label the spatial coordinates. The bars on the various indices in Eq. (1) indicate that this system evolves the coordinate components of the collection of dynamical fields, $u^{\bar{\alpha}}$, as functions of coordinates $x^{\bar{a}}=\left(\bar{t}, x^{\bar{i}}\right)$. In the generalized harmonic system, for example, $u^{\bar{\alpha}}=\left\{\psi_{\bar{a} \bar{b}}, \Pi_{\bar{a} \bar{b}}, \Phi_{\bar{k} \bar{a} \bar{b}}\right\}$ where $\psi_{\bar{a} \bar{b}}$ is the four-metric, $\Pi_{\bar{a} \bar{b}}=-t^{\bar{c}} \partial_{\bar{c}} \psi_{\bar{a} \bar{b}}, \Phi_{\bar{k} \bar{a} \bar{b}}=$ $\partial_{\bar{k}} \psi_{\bar{a} \bar{b}}$, and $t^{\bar{c}}$ is the unit timelike normal to the $\bar{t}=$ const hypersurfaces. We use Latin indices from the beginning of the alphabet, $\bar{a}, \bar{b}, \bar{c}, \ldots$ to label spacetime coordinates.

Einstein's equations are covariant, so it is straightforward to transform any representation of those equations from one coordinate frame to another. The standard method of transformation changes both the coordinates, $x^{\bar{a}} \rightarrow x^{a}$, and the components of the dynamical fields, $u^{\bar{\alpha}} \rightarrow u^{\alpha}$, using the appropriate transformation rules. For the generalized harmonic system, for example, the field components transform according to the rules:

$$
\begin{gathered}
\psi_{a b}=\frac{\partial x^{\bar{a}}}{\partial x^{a}} \frac{\partial x^{\bar{b}}}{\partial x^{b}} \psi_{\bar{a} \bar{b}}, \\
\Pi_{a b}=\frac{\partial x^{\bar{a}}}{\partial x^{a}} \frac{\partial x^{\bar{b}}}{\partial x^{b}} \Pi_{\bar{a} \bar{b}}-2 \frac{\partial^{2} x^{\bar{a}}}{\partial x^{a} \partial x^{c}} \frac{\partial x^{\bar{b}}}{\partial x^{b}} \frac{\partial x^{c}}{\partial x^{\bar{c}}} \psi_{\bar{a} \bar{b}} t^{\bar{c}}, \\
\Phi_{k a b}=\frac{\partial x^{\bar{k}}}{\partial x^{k}} \frac{\partial x^{\bar{a}}}{\partial x^{a}} \frac{\partial x^{\bar{b}}}{\partial x^{b}} \Phi_{\bar{k} \bar{a} \bar{b}}+2 \frac{\partial^{2} x^{\bar{a}}}{\partial x^{k} \partial x^{a}} \frac{\partial x^{\bar{b}}}{\partial x^{b}} \psi_{\bar{a} \bar{b}} .
\end{gathered}
$$

Here we propose a new way of solving the Einstein equations that solves both the rotating frame instability problem and the moving-excision-boundary problems discussed in Sec. I. We introduce a second coordinate system $x^{a}$ that is (in principle) completely independent of the first $x^{\bar{a}}$. We think of the first coordinate system $x^{\bar{a}}$ as inertial coordinates that do not rotate with respect to the asymptotic inertial frame at spatial infinity. We use these inertial coordinate bases, $\partial_{\bar{a}}$ and $d x^{\bar{a}}$, to construct the components of the various dynamical fields: $u^{\bar{\alpha}}$. These inertial-frame components are well behaved near spatial infinity, and the numerical dynamical range needed to represent them is significantly reduced. We think of the second set of coordinates, $x^{a}$, as comoving coordinates chosen to minimize the time dependence of the dynamical fields in some way. We solve the evolution system for the inertial-frame dynamical-field components $u^{\bar{\alpha}}$ as functions of the comoving coordinates $x^{a}$. For simplicity we consider only the case where the two coordinate systems have the same time slicing: $t=\bar{t}$. In this case the system of evolution equations for $u^{\bar{\alpha}}$ in terms of $x^{a}$ is just Eq. (1) with the straightforward change of independent variables: 


$$
\partial_{t} u^{\bar{\alpha}}+\left[\frac{\partial x^{i}}{\partial \bar{t}} \delta^{\bar{\alpha}}{ }_{\bar{\beta}}+\frac{\partial x^{i}}{\partial x^{\bar{k}}} A^{\bar{k}} \bar{\alpha}\right] \partial_{i} u^{\bar{\beta}}=F^{\bar{\alpha}} .
$$

Here $\partial x^{i} / \partial \bar{t}$ and $\partial x^{i} / \partial x^{\bar{k}}$ are to be determined as functions of $t$ and $x^{i}$ from the transformation that relates the two coordinate systems: $x^{i}=x^{i}\left(\bar{t}, x^{\bar{k}}\right)$.

The dual-coordinate evolution system, Eq. (5), has many properties in common with the original, Eq. (1). Its solutions are the same (assuming the coordinate transformation is sufficiently smooth), just expressed in terms of the new coordinates $x^{a}: u^{\bar{\alpha}}\left(x^{a}\right)=u^{\bar{\alpha}}\left[x^{\bar{b}}\left(x^{a}\right)\right]$. In addition, the characteristic fields are exactly the same for the two systems, and the characteristic speeds differ only by $v=\bar{v}+$ $n_{i} \partial x^{i} / \partial \bar{t}$, where $\bar{v}$ represents any of the characteristic speeds of Eq. (1) and $v$ is the corresponding characteristic speed of Eq. (5). Here $n_{i}$ is the appropriate normal oneform used to define the characteristic speeds (e.g., the outward-directed normal to the boundary surface). Thus, boundary conditions for the two systems are transformed in the obvious way. Since the characteristic speeds may be different, however, it is possible that the list of characteristic fields needing boundary conditions at a particular point may change.

\section{ROTATING-COORDINATE FRAMES}

In this section we present a simple test of the dualcoordinate-frame idea described in Sec. II. We evolve asymptotically flat spacetimes, like the Minkowski and Schwarzschild geometries, using two frames: an inertial coordinate frame that is asymptotically Cartesian at infinity, and a comoving coordinate frame that rotates uniformly with respect to the inertial frame.

Before we discuss those tests, however, it is appropriate to describe the numerical methods that we use. These tests are done with the generalized harmonic evolution system as described in Ref. [5] using spectral numerical methods as described, for example, in Refs. [19,20]. The components of the various fields in these tests are expanded in terms of scalar spherical harmonics of the angular coordinates (with $\ell \leq 11$ ) and Chebyshev polynomials of the radial coordinate $\log r$ through order $N_{r}-1$ (with $N_{r}=$ 15 for the tests in Fig. 1). The computational domain used in these tests (as well as those shown in Fig. 1) consists of a set of eight nested spherical shells with boundaries located at the radii 1.8, 8, 35, 70, 140, 229, 374, 612, and 1000M respectively. We measure constraint violations in these tests with a quantity $\|\mathcal{C}\|$ defined as the ratio of the $L^{2}$ norm of all the constraint fields of the generalized harmonic system, divided by the $L^{2}$ norm of the spatial gradients of the various dynamical fields $\partial_{k} u^{\bar{\alpha}}$ (see Eq. [71] of Ref. [5]). This quantity $\|\mathcal{C}\|$ vanishes whenever the constraints are satisfied, and the normalization is chosen so that $\|\mathcal{C}\|$ becomes of order unity when constraint violations dominate the solution.
In $3+1$ formulations of general relativity, the gauge freedom in the theory is usually parametrized by a lapse function and a shift vector that are freely specifiable. In contrast, the gauge freedom in generalized harmonic formulations of Einstein's equations is represented by four freely specifiable gauge source functions $H_{a}$ (see Ref. [5]) which determine the evolution of the lapse and shift. Once $H_{a}$ has been chosen, the harmonic constraint equation

$$
0=\mathcal{C}_{a} \equiv \Gamma_{a}+H_{a}
$$

must be satisfied. Here $\Gamma_{a} \equiv \psi^{b c} \Gamma_{a b c}$ is a trace of the Christoffel symbols. For single-coordinate-frame numerical tests that aim to reproduce a known analytic timeindependent solution, we typically choose $H_{a}$ so that the constraint, Eq. (6), is satisfied initially, and we fix this $H_{a}$ for all time; this was done, for example, in the evolution shown in Fig. 1.

For dual-frame evolutions, we must be more careful with the gauge source functions, particularly because $H_{a}$ does not transform like a tensor. Because we have had considerable success (in nonrotating frames) choosing $H_{a}$ to be time independent, we take a similar approach here. We first define a new quantity $\tilde{H}_{a}$ that has the following two properties: (1) $\tilde{H}_{a}$ transforms like a tensor, and (2) in inertial coordinates $\tilde{H}_{\bar{a}}=H_{\bar{a}}$. As in the single-frame case, we choose $H_{a}$ so that the constraint Eq. (6) is satisfied initially, but now we demand that $\tilde{H}_{a}$ is constant in the moving frame, i.e., that $\partial_{t} \tilde{H}_{a}=0$.

Our first test of the dual-coordinate-frame idea consists of evolving Schwarzschild initial data with uniformly rotating coordinates. In particular, we repeat the most unstable evolution shown in Fig. 1 as a dual-coordinate-frame evolution. The inertial coordinates $(\bar{t}, \bar{x}, \bar{y}, \bar{z})$ for this test are the standard asymptotically Cartesian coordinates associated with the Kerr-Schild representation of the Schwarzschild geometry, while the "comoving" coordinates $(t, x, y, z)$ rotate uniformly with respect to these inertial coordinates:

$$
\begin{gathered}
t=\bar{t}, \\
x=\bar{x} \cos (\Omega \bar{t})+\bar{y} \sin (\Omega \bar{t}), \\
y=-\bar{x} \sin (\Omega \bar{t})+\bar{y} \cos (\Omega \bar{t}), \\
z=\bar{z} .
\end{gathered}
$$

(The angular velocity, $\Omega$, of these comoving coordinates can be chosen arbitrarily.) We solve the dual-coordinate form of the evolution equations, Eq. (5), for these Schwarzschild initial data with $\Omega=0.2 / M$. This value of $\Omega$ is chosen because it corresponds roughly to the orbital angular velocity of a binary black-hole system at the time of merger. Figure 2 shows the constraint violations for these evolutions for several values of the radial resolution parameter $N_{r}$. These dual-coordinate-frame evolutions 


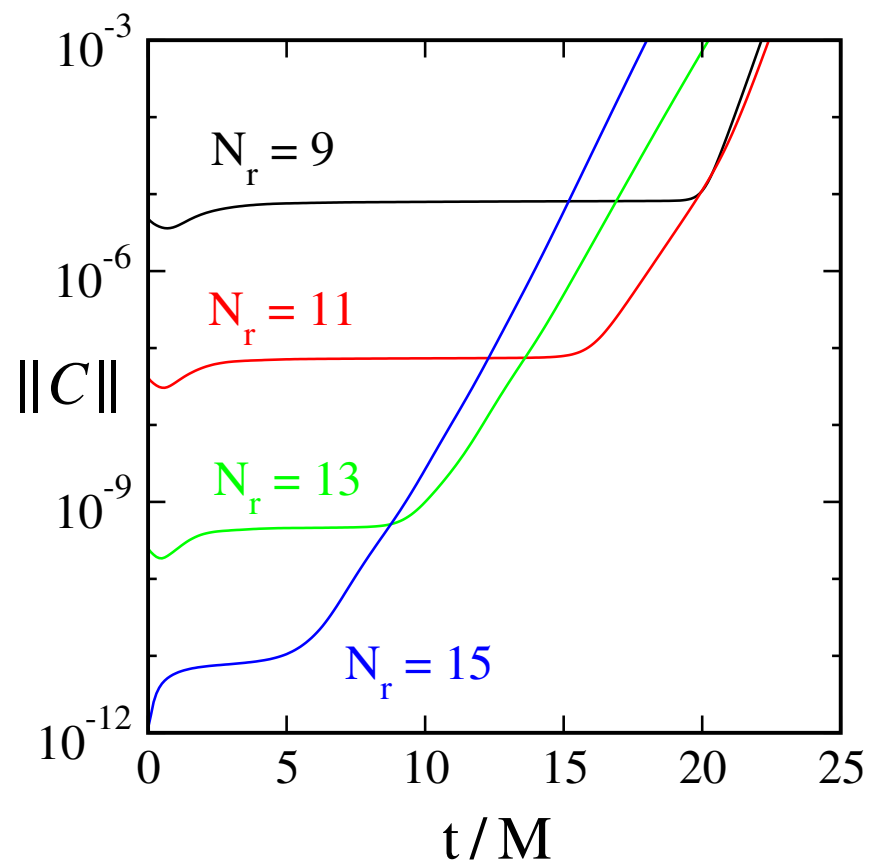

FIG. 2 (color online). Constraint violations in dual-coordinateframe evolutions of Schwarzschild, with comoving coordinates that rotate uniformly with angular velocity $\Omega=0.2 / M$. Angular filtering (see text) is performed on the inertial-frame components.

persist for about $20 M, 5$ orders of magnitude longer than the analogous single coordinate evolution shown in Fig. 1. Unfortunately these evolutions are still unstable on a time scale that is several orders of magnitude shorter than needed, and this instability shows some signs of nonconvergence.

The dual-coordinate-frame representation of tensor fields has some unpleasant features that are ultimately responsible for the instability seen in Fig. 2. If the transformation between the two coordinate frames $x^{i}=x^{i}\left(\bar{t}, x^{\bar{J}}\right)$ is time dependent (as is the case for uniform rotation), then clearly the transformation relating the tensor bases, e.g., $\partial_{\bar{\imath}}=\partial x^{j} / \partial x^{\bar{l}} \partial_{j}$, will also be time dependent. So, even if the comoving components of a tensor $\psi_{a b}\left(x^{j}\right)$ are time independent, as is the case for the Schwarzschild example above, then the inertial-frame components $\psi_{\bar{a} \bar{b}}\left(t, x^{j}\right)$ that we are evolving will change with time. Consider, for example, a purely radial vector field $v^{i}\left(x^{j}\right)$ that is time independent when represented in a single coordinate frame that rotates with angular velocity $\Omega$. Figure 3 illustrates the time dependence of the inertial-frame components $v^{\bar{i}}\left(x^{j}\right)$ of this vector field. Two cross sections of this field (one equatorial and one polar) are shown at time $t=0$, when the rotation map is just the identity, and at times $t=\pi /(2 \Omega)$ and $t=\pi / \Omega$, when each inertial-frame basis vector $\partial_{\bar{l}}$ is rotated by $\pi / 2$ and $\pi$ about the $z$ axis, respectively.

In addition to making tensor field components time dependent, the dual-coordinate-frame representation can

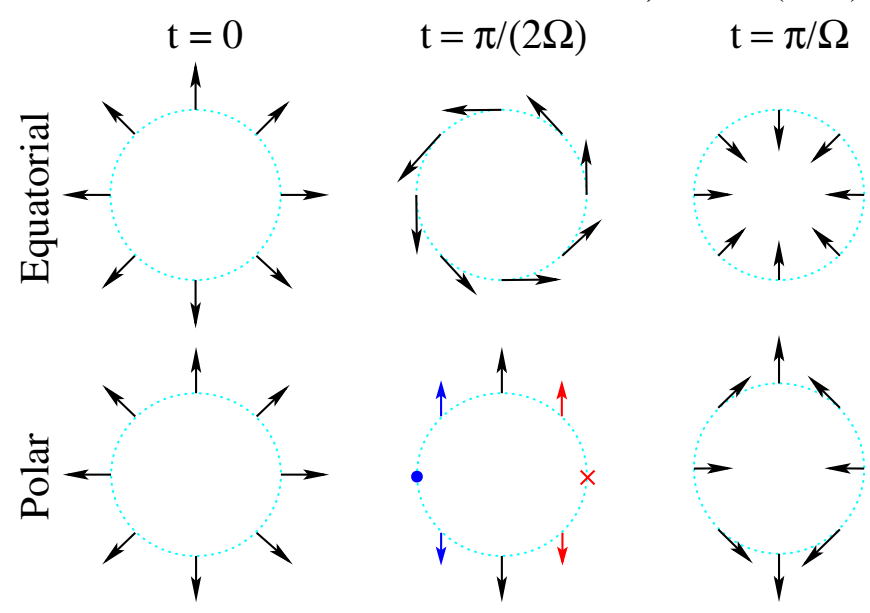

FIG. 3 (color online). Time dependence of the inertial-frame components of two cross sections (equatorial and polar) of a vector field expressed as functions of rotating coordinates. At $t=0$ the vector field is radial, but the $\partial_{\bar{x}}$ and $\partial_{\bar{y}}$ basis vectors rotate with angular velocity $\Omega$. The vectors on the right side of the polar cross section figure at $t=\pi /(2 \Omega)$ are pointed into the plane of the figure, while those on the left point out.

also mix the tensor-spherical-harmonic components of tensors. In Fig. 3, for example, the radial vector field is pure $\ell=0$ when represented in rotating-frame vector spherical harmonics, but it becomes a time-dependent mixture of $\ell=0,1$, and 2 when represented in inertialframe vector spherical harmonics. Figure 4 illustrates the time dependence of the tensor-spherical-harmonic components of the Schwarzschild spatial three-metric expressed in rotating coordinates. The dashed curves are the rotating-frame tensor-spherical-harmonic components, while the solid curves are the inertial-frame tensor-spherical-harmonic components. In the rotating frame, the Schwarzschild spatial three-metric is pure $\ell=0$, while the inertial-frame components are a time-dependent mixture of $\ell=0,1,2,3$, and 4 . The periodicity of the tensorspherical-harmonic components in Fig. 4 is determined by the rotation period of the coordinates $2 \pi / \Omega$.

This mixing of the inertial-frame tensor-sphericalharmonic components is the cause of the instability seen in Fig. 2. When using spectral methods in a spherical computational domain, we expand the Cartesian components of tensors in scalar spherical harmonics, and we evolve these Cartesian components. In this case we find it necessary periodically to set to zero the highest-order tensor-spherical-harmonic coefficients of all tensors in order to avoid numerical instabilities [19,21]. We call this operation "filtering," since it is similar to the filtering operations commonly used in spectral methods to avoid instabilities caused by aliasing. Filtering is necessary because spatial differentiation couples different values of the $\ell$ and $m$ indices in the scalar-spherical-harmonic expansions of the Cartesian components of tensors. Consequently, any truncated series expansion will result in 


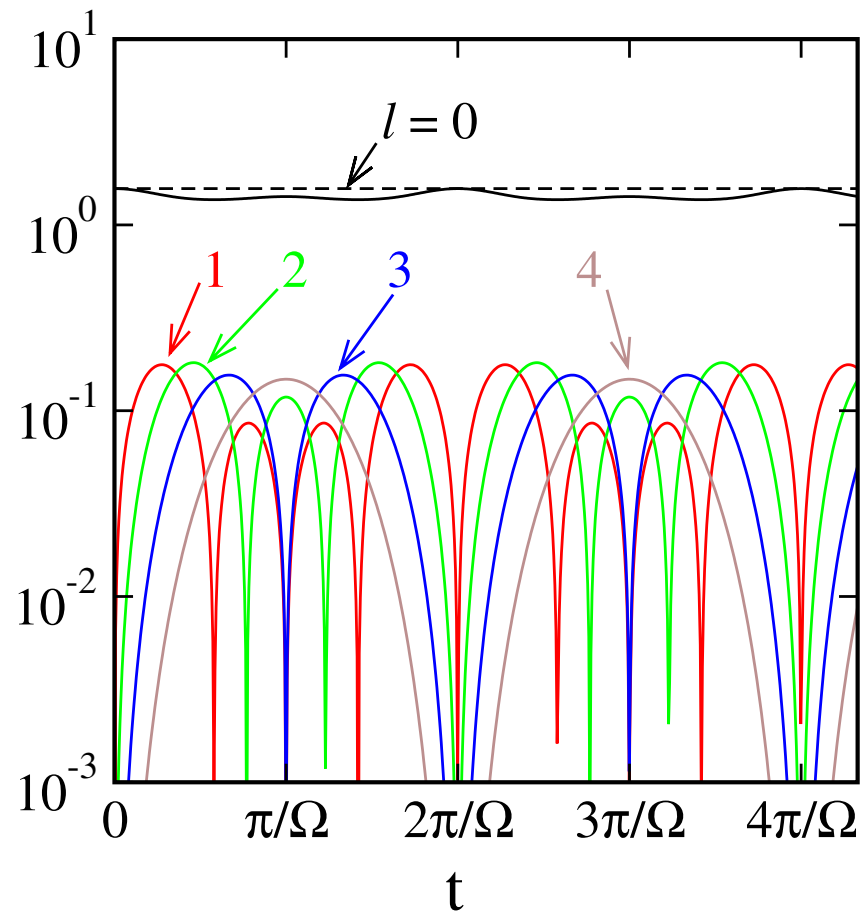

FIG. 4 (color online). Tensor-spherical-harmonic components of the Schwarzschild spatial three-metric expressed in rotating coordinates. Dashed curves show the components expressed in rotating-frame tensor spherical harmonics (only the $\ell=0$ component is nonzero); solid curves show the inertial-frame tensorspherical-harmonic components.

incorrect evolution equations for the highest angular modes, and these errors often lead to nonconvergent instabilities. However, it is possible to truncate the tensorspherical-harmonic expansion of a tensor at a finite value of $\ell$ in a self-consistent way, because the spatial gradient of a tensor spherical harmonic is also a tensor spherical harmonic with the same spherical harmonic index, but one higher tensor rank. Thus, we perform filtering by transforming each tensor from a Cartesian-component basis to a tensor-spherical-harmonic basis, zeroing the tensorspherical-harmonic coefficients for values of $\ell$ larger than those kept in our expansion, and transforming back to our Cartesian-component basis. This filter cures the angular instability problems associated with the evolutions of single-coordinate frame spherical-harmonic representations of tensors $[19,21]$.

This filtering algorithm must be modified for dual-coordinate-frame methods because there is now a more complicated relationship between the coordinates and the basis vectors used to represent tensors. Figures 3 and 4 illustrate that inertial-frame components of a tensor expressed as functions of rotating-frame coordinates have some additional time-dependent mixing among the various tensorspherical-harmonic components. Therefore, filtering the inertial-frame components using the same algorithm used for single-coordinate frame evolutions is the wrong thing to do: it does not preserve all of the information needed to determine a number of the highest-index coefficients in this case. This straightforward (and incorrect) implementation of spherical-harmonic filtering is the method used for the test shown in Fig. 2, with the result being unstable (in the sense that the constraints grow without bound) and probably nonconvergent. The cure for this problem is also clear: transform spatial tensors to a rotating-frame tensorspherical-harmonic basis before filtering, then transform back to the inertial-frame basis afterwards. (Spacetime tensors, such as the four-metric $\psi_{\bar{a} \bar{b}}$, are first split into their spatial-tensor parts, e.g., $\psi_{\bar{t} \bar{t}}, \psi_{\bar{t} \bar{l}}$, and $\psi_{\bar{i} \bar{j}}$, and these parts are then filtered in this way.) Results using this new filtering algorithm are shown in Fig. 5 for the same initial data and dual-coordinate frames used in Fig. 2. These evolutions now appear to be stable and convergent on the needed time scale, except for mild (sublinear) power-law growth seen here only in the highest resolution cases. We suspect that this power-law growth may be due to accumulated roundoff error, but we have not investigated this in detail because the growth is insignificant on the time scale needed for multiple-orbit binary black-hole evolutions such as those described in Sec. V. The new filtering method described here is needed to perform stable evolutions of any rotating spacetime (even "linear" problems like evolutions of Minkowski spacetime) using the dual-frame

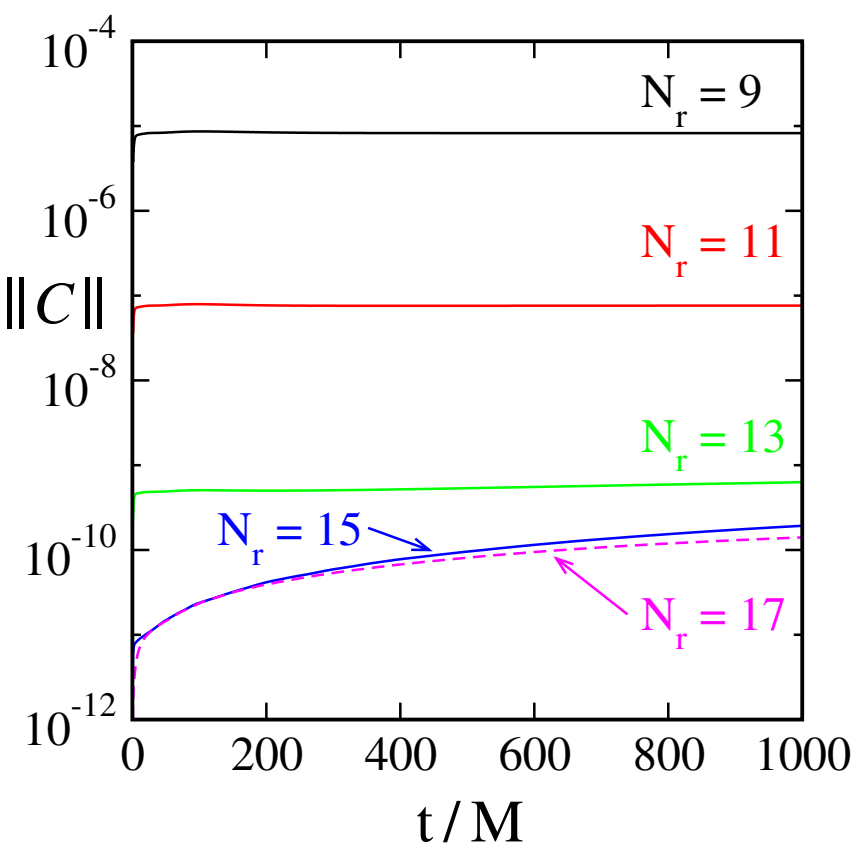

FIG. 5 (color online). Constraint violations in dual-coordinateframe evolutions of Schwarzschild, with comoving coordinates that rotate uniformly with angular velocity $\Omega=0.2 / M$. These evolutions use the new rotating-frame tensor-spherical-harmonic filtering algorithm. The highest resolution case (dashed curve) uses more angular basis functions $(\ell \leq 13$ instead of $\ell \leq 11)$ as a more stringent test of convergence. 
method. Additional filtering may also be needed in some circumstances to control other problems (spectral aliasing errors due to nonlinearities, for example), but no additional filtering was needed or used for any of the numerical evolutions presented in this paper.

\section{BINARY BLACK-HOLE TESTS}

The primary motivation for developing the dualcoordinate method describe in Sec. II was to allow us to perform binary black-hole evolutions using coordinates that move with the holes. In this section we describe the first test of this new method for binary black-hole evolutions. We describe the binary black-hole initial data that we use, give a very brief description of the binary black-hole specific features of our numerical methods, and then describe the results of this first test.

The binary black-hole evolutions described here begin with initial data prepared with the methods described in Ref. [22]. These data represent two equal-mass corotating black holes in a quasistationary circular orbit. The data are obtained by solving the extended conformal thin-sandwich form of the initial value equations with the following choices of freely specifiable data: the spatial conformal metric is chosen to be flat, the trace of the extrinsic curvature, its time derivative, and the time derivative of the conformal metric are set to zero. In addition to determining the spatial metric and extrinsic curvature, this form of the initial value equations also determines a lapse and shift that produce relatively time-independent evolutions. We use the Neumann form of the lapse boundary condition described in Ref. [22]. The binary black holes used for the tests described here have equal irreducible masses, $M_{\text {irr }}=$ 1.061536 (in our code coordinate units), total ADM energy $M_{\mathrm{ADM}}=2.100609=1.978834 M_{\text {irr }}$, and total ADM angular momentum $J_{\mathrm{ADM}}=4.3485850=0.985502 M_{\mathrm{ADM}}^{2}$. The coordinate separation of the centers of these holes is set to 20 initially, and the initial spins of the holes are set to corotating values (i.e., no spin in the corotating frame). The initial orbital angular velocity of this binary is $\Omega=$ $0.01418276=0.02979244 / M_{\mathrm{ADM}}$. This initial data set is from a family of publicly available initial data [23].

We represent the binary black holes considered here on a computational domain divided into 44 subdomains: 14 spherical shells, 24 cylindrical shells, and 6 rectangular blocks. The various dynamical fields are expanded in the appropriate spectral basis functions for each subdomain. The evolutions described here are performed at two numerical resolutions using a total (over all subdomains) of $260756 \approx 64^{3}$ and $431566 \approx 76^{3}$ collocation points, respectively. Constraint preserving and physical boundary conditions are imposed at the outer boundary of our computational domain (initially at $r=280$ ) as described in Ref. [5], and the appropriate characteristic fields are exchanged between subdomains at internal boundaries. A more detailed description of the numerical methods used for these binary black-hole evolutions will be included in a subsequent paper. Here we focus our attention on the dualcoordinate aspects of these evolutions.

As our first binary black-hole test of the dual-coordinate evolution method, we evolve the equal-mass circular-orbit binary described above using a rotating-coordinate frame as described in Sec. III. We set the angular velocity of this frame to $\Omega=0.02979244 / M_{\mathrm{ADM}}$, the initial orbital angular velocity of the binary. We track the position of the center of each black hole, $\left[x_{c}(t), y_{c}(t), z_{c}(t)\right]$, during this evolution by solving for the apparent horizon of each hole. We express the apparent horizon as a spherical-harmonic expansion [24]: $\vec{R}_{\mathrm{AH}}=\vec{c}+\hat{r} \sum_{\ell, m} R_{l m}(t) Y_{l m}(\theta, \varphi)$, where $\vec{c}$ is the point about which we expand, $\hat{r}$ is a radial unit vector field centered on $\vec{c}$, and $\theta$ and $\varphi$ label the points on the apparent-horizon surface. The coefficients $R_{l m}$ are obtained by minimizing the expansion at the GaussLegendre collocation points $[25,26]$. The center of the hole is defined as that $\vec{c}$ for which the $\ell=1$ components of the spherical harmonic expansion vanish: $R_{1 m}=0$. Initially the black holes are located at $\left[x_{c}(0), y_{c}(0)\right.$, $\left.z_{c}(0)\right]=[ \pm 10,0,0]$. The reflection symmetry of the initial data ensures that $z_{c}(t)=0$ throughout the evolution, but $x_{c}(t)$ and $y_{c}(t)$ are free to drift.

Figure 6 illustrates the motion of the center of one of the black holes, $x_{c}(t)$ and $y_{c}(t)$, with respect to the comoving coordinates. We see that the center of the hole remains relatively fixed during the very first part of this evolution,

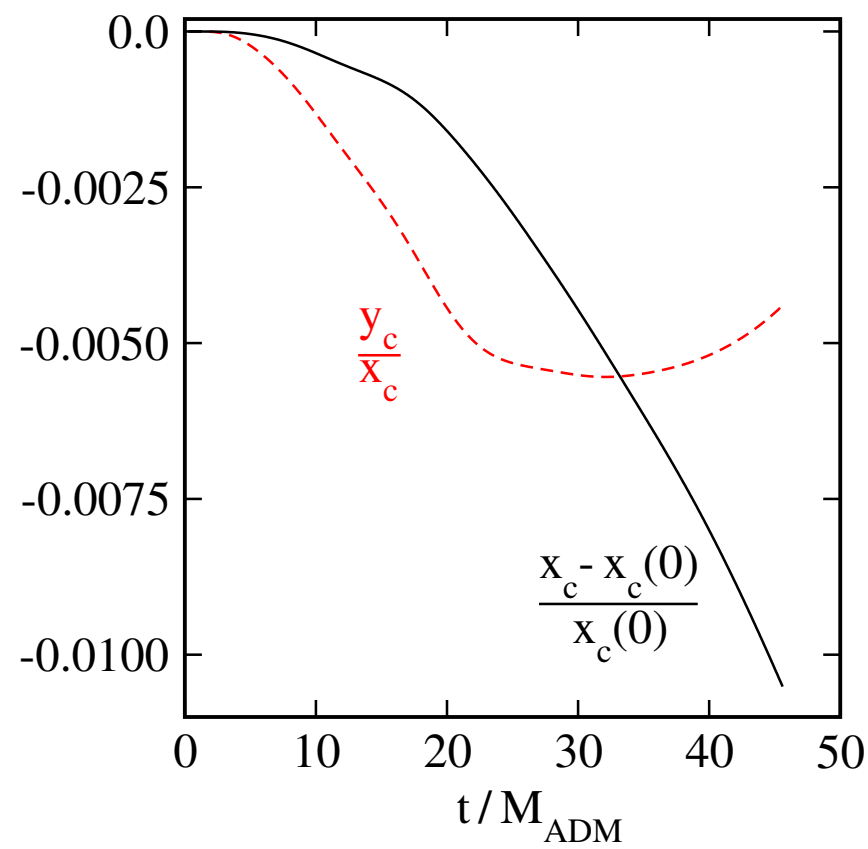

FIG. 6 (color online). Evolution of the apparent horizon center, $\left(x_{c}, y_{c}\right)$, of one of the black holes relative to a uniformly rotatingcoordinate frame. The minimum coordinate distance between the apparent horizon and the excision boundary becomes less than 0.003 at $t=45.6 M_{\mathrm{ADM}}$, and the evolution is terminated. 
but fairly quickly it begins to drift away from its initial location with $x_{c}(t)$ drifting more quickly than $y_{c}(t)$. It appears that the two black holes get closer together due to the effects of gravitational radiation on the orbit. Our code automatically terminates an evolution when an apparent horizon gets too close (within 0.003 for this run) to an inner (excision) boundary of the computational domain. If the apparent horizon were to cross this boundary, then additional boundary conditions would be needed there and we do not know how to specify such boundary conditions in a physically meaningful way.

Figure 7 shows the equatorial ( $x-y$ plane) cross sections of the initial apparent horizon (solid curve), the final apparent horizon (dashed curve), and the location of the excision boundary (dotted curve) for one of the black holes from the evolution shown in Fig. 6. The apparent horizon has shifted to the left, confirming our diagnosis that the two black holes have gotten closer together. Our code does not crash at the end of this test, and the evolution would have run (a little) longer had a smaller excision boundary been used. A smaller excision boundary results in higher truncation errors for a fixed numerical resolution, so making it smaller requires significantly more computational resources. But even if cost were not an issue, making the excision boundary smaller would only delay the apparent horizon crossing the excision boundary by a very brief time. A better solution is needed for this problem.

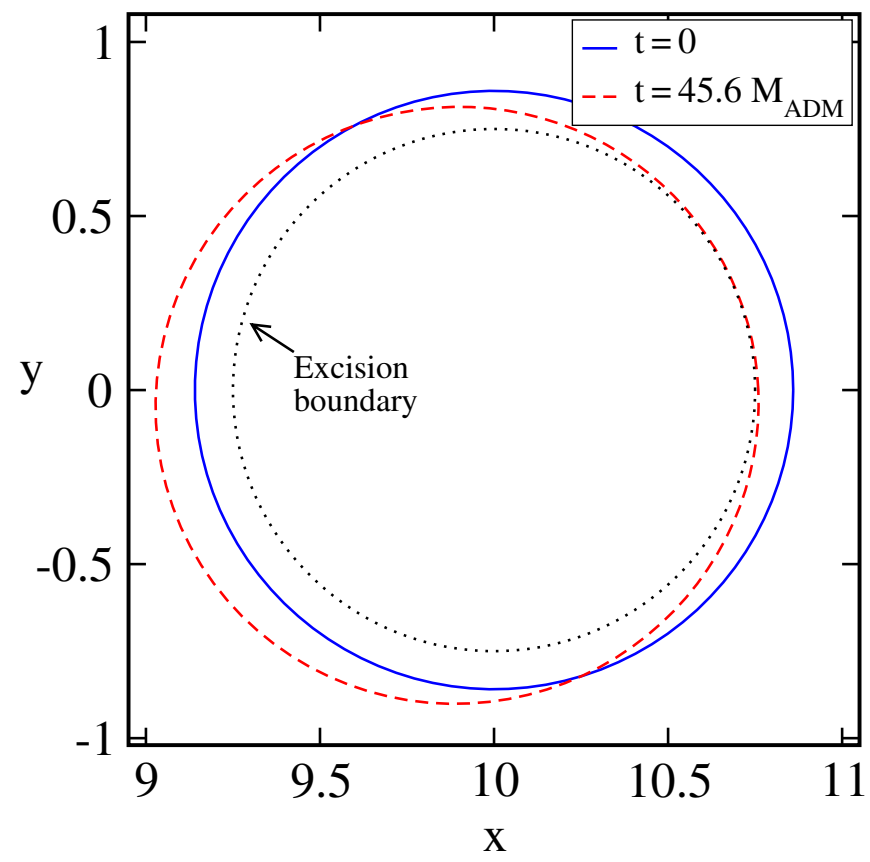

FIG. 7 (color online). Moving coordinate frame representation of an equatorial slice through the apparent horizon of one of the black holes for the evolution of Fig. 6. The evolution is terminated when the apparent horizon approaches the excision boundary (dotted curve) near the right side of this figure.

\section{HORIZON-TRACKING COORDINATES}

The binary black-hole test described in Sec. IV shows that a uniformly rotating comoving coordinate frame is not adequate to keep the black holes centered on the excision boundaries of the computational domain. The motions of the centers of the holes are too time dependent for this simple approach to work effectively for very long. If we wish to construct coordinates that track the apparent horizons for many orbits, then a more flexible coordinate map is needed. Since the positions of the black holes are difficult (impossible) to predict a priori, some kind of feedback control system will also be needed to measure these positions and make the appropriate adjustments to the coordinate maps as the evolution proceeds.

The motion of the black holes in the test of Sec. IV had a larger $x_{c}(t)$ component, so we begin by constructing a coordinate map that more accurately tracks this component of the black hole's position. Let us define a control parameter, $Q^{x}(t) \equiv\left[x_{c}(t)-x_{c}(0)\right] / x_{c}(0)$, that gives a dimensionless measure of the $x$-component of the position of the hole. The idea is to measure $Q^{x}(t)$ as the binary evolves, and then use this information to adjust the function $a(t)$ in the time-dependent coordinate map,

$$
\begin{gathered}
t=\bar{t} \\
x=a(\bar{t})[\bar{x} \cos (\Omega \bar{t})+\bar{y} \sin (\Omega \bar{t})], \\
y=a(\bar{t})[-\bar{x} \sin (\Omega \bar{t})+\bar{y} \cos (\Omega \bar{t})], \\
z=a(\bar{t}) \bar{z}
\end{gathered}
$$

in such a way that $Q^{x}(t)$ remains sufficiently small. This map applies the uniform rotation used in Sec. IV combined with a rescaling of the spatial coordinates that can be used to keep the $x$-coordinate separation of the holes fixed.

We borrow ideas from the literature on mathematical control theory [27] to design a feedback control system for the map parameter $a$. The basic idea is to change $a$ in such a way that $Q^{x}$ is driven back to its "equilibrium" value, $Q^{x}=0$, whenever it drifts away. The first step is to determine the response of the control parameter $\delta Q^{x}$ to a small change in the map parameter $\delta a$. In this case the relationship is rather simple: $\delta Q^{x}=-\delta a / a$. For maps that are close to the identity, $a=1$, this relationship is well approximated by $\delta Q^{x} \approx-\delta a$. We also want to make sure that any adjustment we make to the coordinate map is sufficiently smooth that it does not interfere with our ability to solve the Einstein equations. In particular, we need the coordinate map to be at least $C^{2}$ so that the transformed dynamical fields $u^{\bar{\alpha}}$ are at least continuous [see Eqs. (3)-(5)]. Therefore our control system will be allowed to adjust only $d^{3} a / d t^{3}$ freely, and $a$ will be obtained by time integration to ensure sufficient smoothness. Following the usual procedure in control theory, we choose $d^{3} a / d t^{3}$ in a way that is determined by the measured values 
of $Q^{x}$ and its derivatives:

$$
\frac{d^{3} a}{d t^{3}}=\alpha Q^{x}+\beta \frac{d Q^{x}}{d t}+\gamma \frac{d^{2} Q^{x}}{d t^{2}},
$$

where $\alpha, \beta$, and $\gamma$ are parameters that we are free to pick. We choose these parameters in such a way that the "closed-loop" equation,

$$
\frac{d^{3} a}{d t^{3}}=-\frac{d^{3} Q^{x}}{d t^{3}}=\alpha Q^{x}+\beta \frac{d Q^{x}}{d t}+\gamma \frac{d^{2} Q^{x}}{d t^{2}},
$$

has solutions that all decay exponentially toward the desired equilibrium value $Q^{x} \rightarrow 0$. We use the values $\alpha=$ $\lambda^{3}, \beta=3 \lambda^{2}$, and $\gamma=3 \lambda$, that result in the following closed-loop equation:

$$
\frac{d^{3} Q^{x}}{d t^{3}}=-\lambda^{3} Q^{x}-3 \lambda^{2} \frac{d Q^{x}}{d t}-3 \lambda \frac{d^{2} Q^{x}}{d t^{2}} .
$$

The most general solution to this equation is

$$
Q^{x}(t)=\left(A t^{2}+B t+C\right) e^{-\lambda t},
$$

for arbitrary constants $A, B$, and $C$. All of these solutions decay exponentially toward the desired equilibrium solution at a rate determined by the parameter $\lambda>0$.

We use these ideas now to construct a feedback control system for $a$. We pick a set of control times $t_{i}$ at which the expression for $a$ will be adjusted. The control interval $\Delta t=t_{i+1}-t_{i}$ is chosen to be shorter than the time scale on which $Q^{x}$ drifts away from its equilibrium value. We choose the map parameter $a(t)$ in the time interval $t_{i} \leq$ $t<t_{i+1}$ to be

$$
\begin{aligned}
a(t)= & a_{i}+\left(t-t_{i}\right) \frac{d a_{i}}{d t}+\frac{\left(t-t_{i}\right)^{2}}{2} \frac{d^{2} a_{i}}{d t^{2}}+\frac{\left(t-t_{i}\right)^{3}}{2} \\
& \times\left(\lambda \frac{d^{2} Q_{i}^{x}}{d t^{2}}+\lambda^{2} \frac{d Q_{i}^{x}}{d t}+\lambda^{3} \frac{Q_{i}^{x}}{3}\right),
\end{aligned}
$$

where the constants $a_{i}, d a_{i} / d t$, and $d^{2} a_{i} / d t^{2}$ are the values taken from the map in the previous interval $t_{i-1} \leq t<t_{i}$ evaluated at $t=t_{i}$, and the constants $Q_{i}^{x}, d Q_{i}^{x} / d t$, and $d^{2} Q_{i}^{x} / d t^{2}$ are the values measured at $t=t_{i}$. This choice of $a(t)$ guarantees that our comoving coordinate map is $C^{2}$ in time, $C^{\infty}$ in space, and it enforces the closed-loop equation exactly at the control times $t=t_{i}$. We begin our evolutions at $t_{0}=0$ by setting the initial conditions $a_{0}=1$ and $d a_{0} / d t=d^{2} a_{0} / d t^{2}=Q_{0}^{x}=d Q_{0}^{x} / d t=d^{2} Q_{0}^{x} / d t^{2}=$ 0 . To ensure that the discontinuities in $d^{3} a / d t^{3}$ do not affect the convergence of our code, we require that the $t_{i}$ occur at fixed times (independent of the numerical time step), and we choose the time steps so that the $t_{i}$ always coincide with the beginning of a full numerical time step.

We test this feedback control system by evolving the same binary black-hole initial data discussed in Sec. IV. We set the value of the control damping parameter $\lambda \approx$ $0.3 / M_{\mathrm{ADM}}$, the control interval $\Delta t \approx 1 M_{\mathrm{ADM}}$, and the time scale used to evaluate average values of the derivatives of
$Q^{x}$ in Eq. (19) to $\sim 0.5 M_{\mathrm{ADM}}$. Figure 8 shows the motion of the center of one black hole during this test. We see that the control system effectively keeps the $x$-coordinate of the center of the hole $Q^{x}=\left[x_{c}-x_{c}(0)\right] / x_{c}(0)$ within acceptable bounds. But this test ends at about $t=73.4 M_{\mathrm{ADM}}$ when the other (uncontrolled) component of the black hole's position $y_{c} / x_{c}$ grows too large. The new control system significantly extends this binary evolution, but the binary has still only completed about 0.34 orbits. Figure 9 shows that the apparent horizon has drifted upward at the time this test ends. As the binary inspirals towards merger the angular velocity of the orbit increases, so the fixed angular velocity of the comoving coordinates is no longer able to track the positions of the holes with sufficient accuracy.

Fortunately, the dual-coordinate evolution method is extremely flexible and it is easy to construct a comoving coordinate map capable of tracking both the orbital rotation and the radial motion of the black holes. For example, the map

$$
t=\bar{t}
$$

$$
\begin{gathered}
x=a(\bar{t})[\bar{x} \cos \varphi(\bar{t})+\bar{y} \sin \varphi(\bar{t})], \\
y=a(\bar{t})[-\bar{x} \sin \varphi(\bar{t})+\bar{y} \cos \varphi(\bar{t})] \\
z=a(\bar{t}) \bar{z}
\end{gathered}
$$

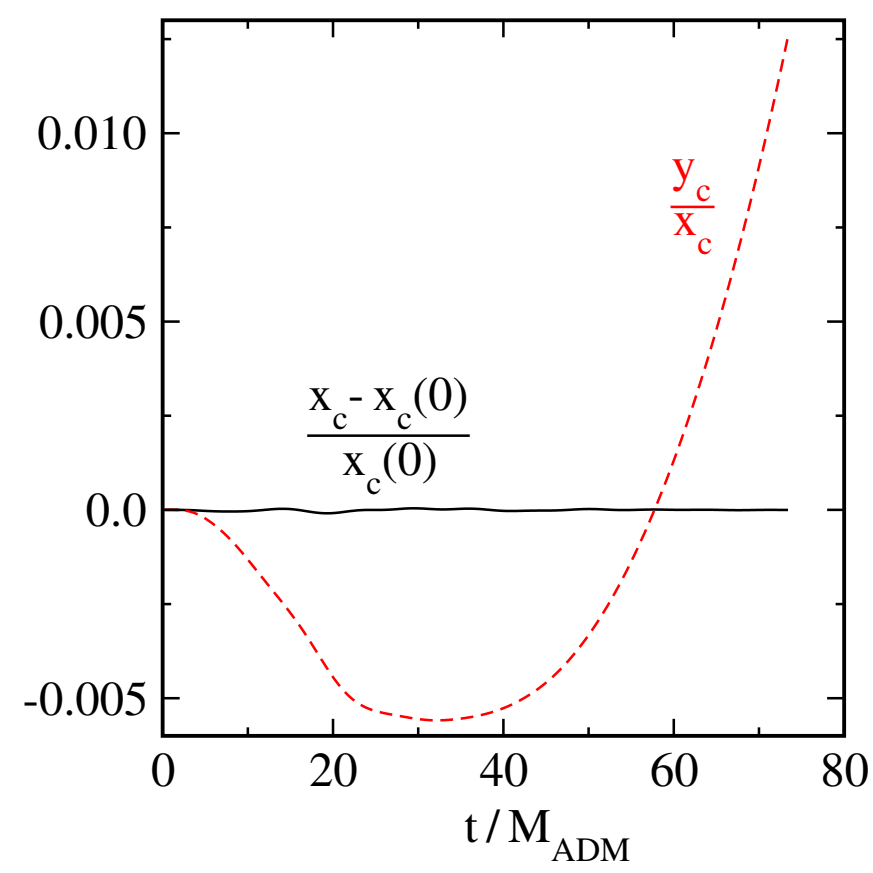

FIG. 8 (color online). Evolution of the apparent horizon center of one of the holes for a binary black-hole evolution using a feedback control system that adjusts the moving coordinate frame to control the parameter $\left[x_{c}-x_{c}(0)\right] / x_{c}(0)$. Compare to Fig. 6. 


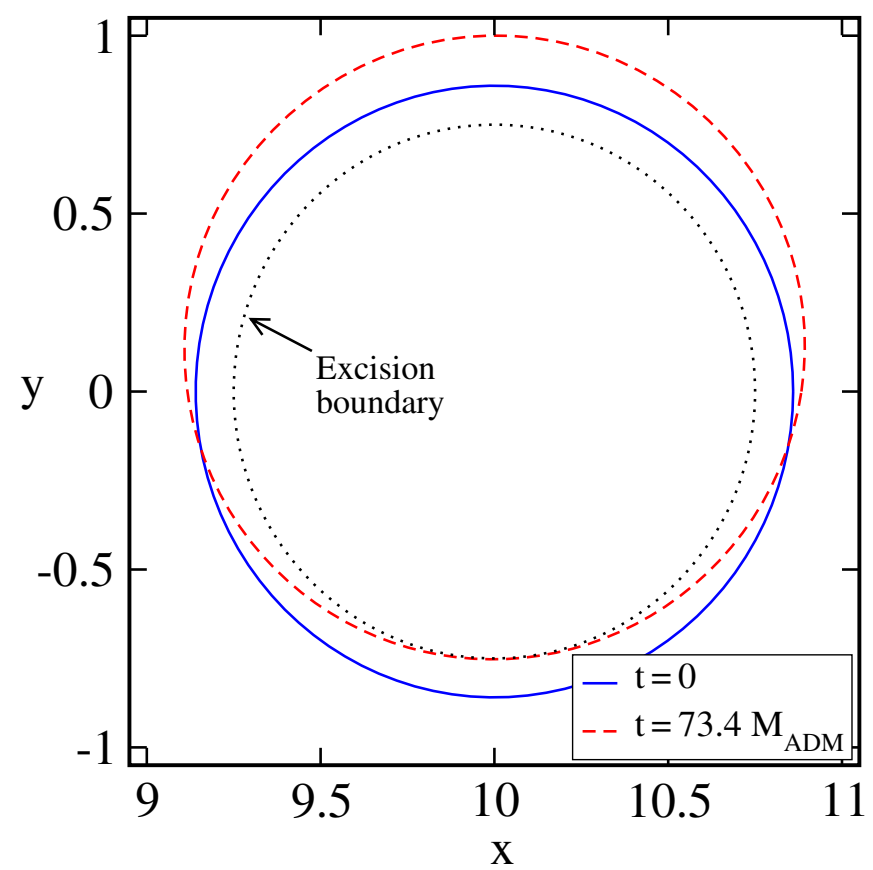

FIG. 9 (color online). Moving coordinate frame representation of an equatorial slice through the apparent horizon of one of the black holes for the evolution of Fig. 8. Evolution is terminated when the apparent horizon approaches the excision boundary near the bottom of this figure.

includes a time-dependent rotation angle $\varphi(t)$ that can be used to adjust the angular velocities of the holes, in addition to the time-dependent conformal factor $a(t)$. An additional feedback control system is then needed to adjust the map parameter $\varphi$ through measurements of the control parameter $Q^{y} \equiv y_{c} / x_{c}$. The basic control equation for this parameter is $\delta Q^{y}=-\delta \varphi$. So we construct a feedback control system for $\varphi(t)$ that is completely analogous to Eq. (19). In the time interval $t_{i} \leq t<t_{i}$ we set

$$
\begin{aligned}
\varphi(t)= & \varphi_{i}+\left(t-t_{i}\right) \frac{d \varphi_{i}}{d t}+\frac{\left(t-t_{i}\right)^{2}}{2} \frac{d^{2} \varphi_{i}}{d t^{2}}+\frac{\left(t-t_{i}\right)^{3}}{2} \\
& \times\left(\lambda \frac{d^{2} Q_{i}^{y}}{d t^{2}}+\lambda^{2} \frac{d Q_{i}^{y}}{d t}+\lambda^{3} \frac{Q_{i}^{y}}{3}\right),
\end{aligned}
$$

where the constants $\varphi_{i}, d \varphi_{i} / d t$, and $d^{2} \varphi_{i} / d t^{2}$ are the values taken from the map in the previous interval $t_{i-1} \leq$ $t<t_{i}$ evaluated at $t=t_{i}$, and the constants $Q_{i}^{y}, d Q_{i}^{y} / d t$, and $d^{2} Q_{i}^{y} / d t^{2}$ are the values measured at $t=t_{i}$. This choice of $\varphi(t)$ guarantees that our comoving coordinate map is $C^{2}$, and it enforces the closed-loop equation periodically at the control times $t=t_{i}$. We begin our evolutions at $t_{0}=0$ by setting the initial conditions $d \varphi_{0} / d t=\Omega \quad$ and $\quad \varphi_{0}=d^{2} \varphi_{0} / d t^{2}=Q_{0}^{y}=d Q_{0}^{y} / d t=$ $d^{2} Q_{0}^{y} / d t^{2}=0$.
We test this enhanced feedback control system, which controls both $Q^{x}$ and $Q^{y}$, by evolving the same binary black-hole initial data used for the evolutions in Figs. 6 and 8 . We use the same control system parameters for the $Q^{x}$ subsystem as those discussed above. For the $Q^{y}$ subsystem, we set the value of the control damping parameter $\lambda \approx 0.5 / M_{\mathrm{ADM}}$, the control interval $\Delta t \approx 0.5 M_{\mathrm{ADM}}$, and the time scale used to evaluate the average values of the derivatives of $Q^{y}$ in Eq. (24) to $\sim 0.2 M_{\mathrm{ADM}}$. Figure 10 shows the control parameters $Q^{x}$ and $Q^{y}$ for this evolution. We see that both control systems work extremely well, and the binary now evolves until $t=661.4 M_{\mathrm{ADM}}$ before the evolution stops. Figure 11 shows the evolution of the inertial-frame coordinates $\left[\bar{x}_{c}(\bar{t}), \bar{y}_{c}(\bar{t})\right]$ of the center of one of the black holes, from which we see that this evolution has completed about 4.6 orbits. Figure 12 shows the normalized constraint violations for this evolution, at two different numerical resolutions. The upper curve uses a total of $260756 \approx 64^{3}$ collocation points and the lower curve uses $431566 \approx 76^{3}$ collocation points. The constraints are normalized here by dividing by the initial value of the norm of the derivatives of the dynamical fields. Figure 12 suggests that our numerical evolutions are convergent until just before the code terminates.

Finally, Fig. 13 shows the shape of the apparent horizon of one black hole (expressed in comoving coordinates) at $t=0$ (solid curve) and at the end of the evolu-

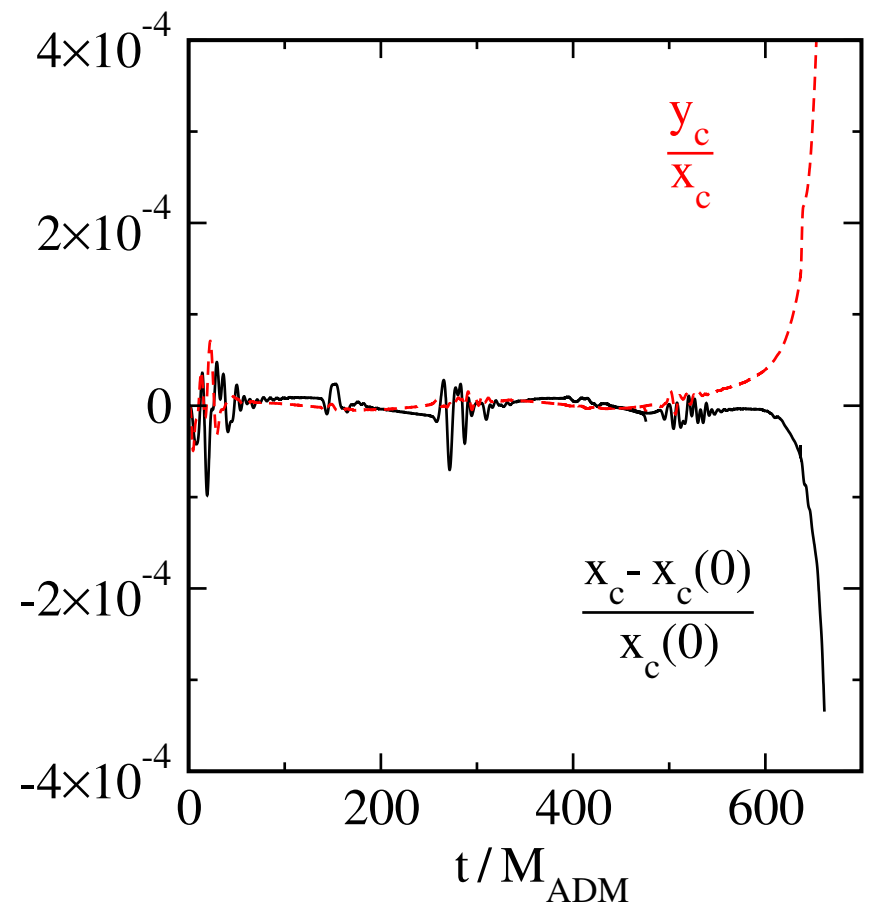

FIG. 10 (color online). Evolution of the apparent horizon center of one black hole for a binary black-hole evolution using a feedback system that adjusts the moving coordinate frame to control both the parameters $y_{c} / x_{c}$ and $\left[x_{c}-x_{c}(0)\right] / x_{c}(0)$. Compare to Figs. 6 and 8. 


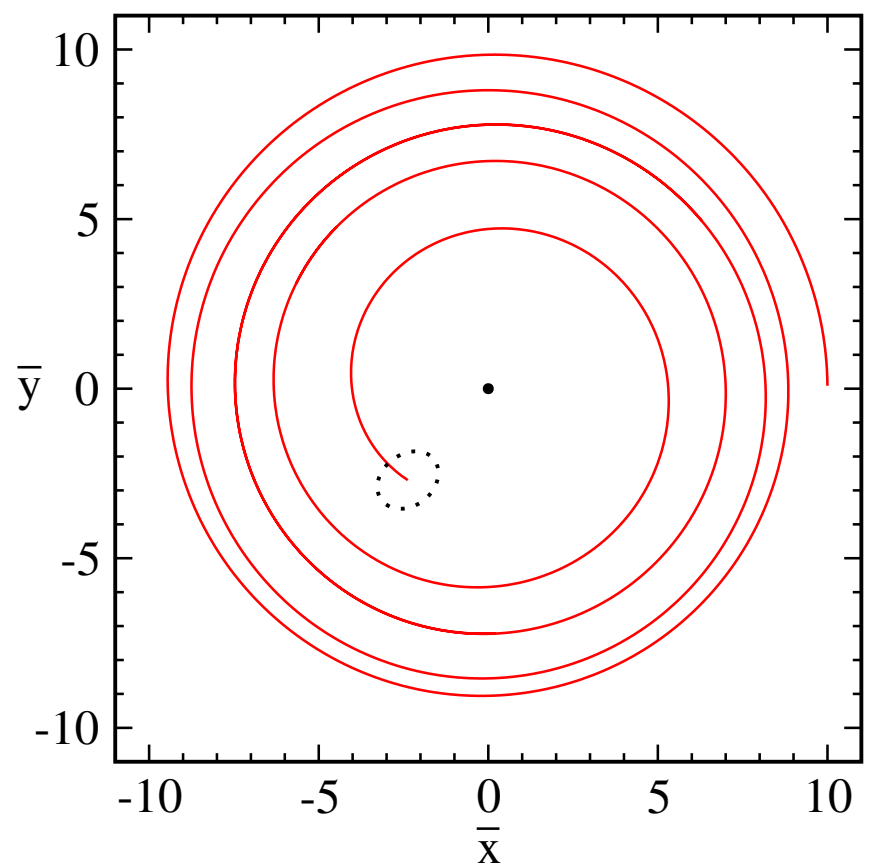

FIG. 11 (color online). Motion of the inertial-frame coordinates of the center of the apparent horizon $\left(\bar{x}_{c}, \bar{y}_{c}\right)$ (solid curve) of one of the black holes from the evolution of Fig. 10. The dotted curve shows the equatorial cross section of the apparent horizon at $t=661.4 M_{\mathrm{ADM}}$. The binary system has completed about 4.6 orbits.

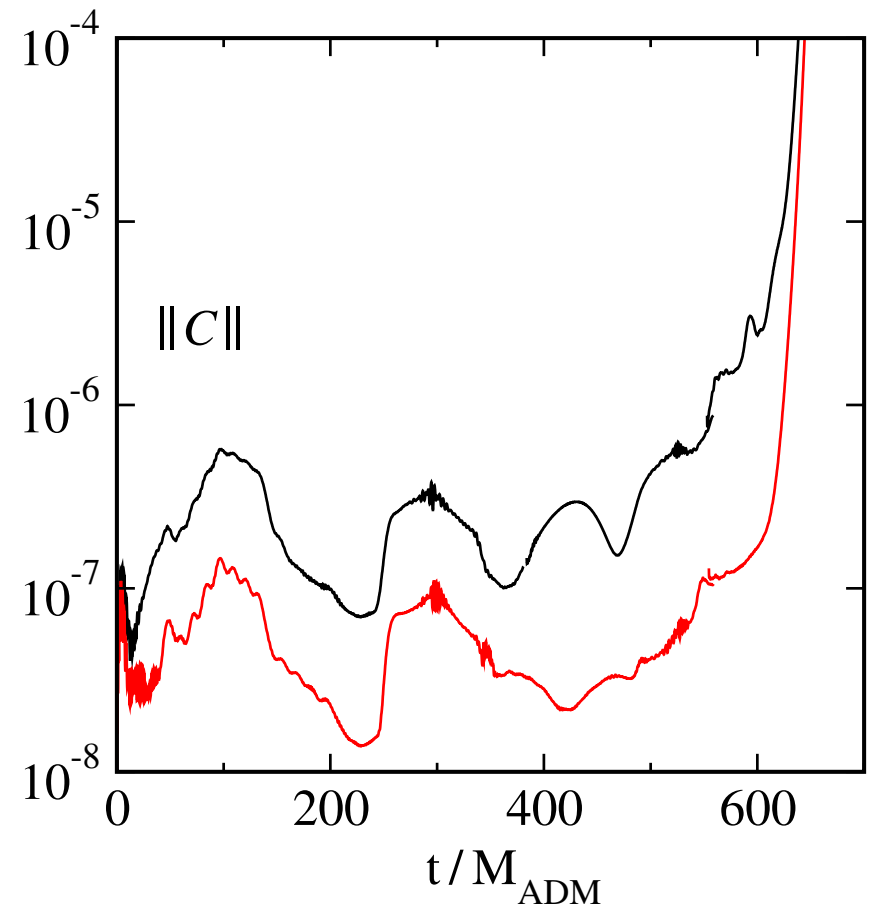

FIG. 12 (color online). Constraint violations $\|\mathcal{C}\|$ for the binary black-hole evolution of Fig. 10. The two curves represent two numerical resolutions, the upper curve has about $64^{3}$ collocation points, while the lower curve has about $76^{3}$ collocation points.

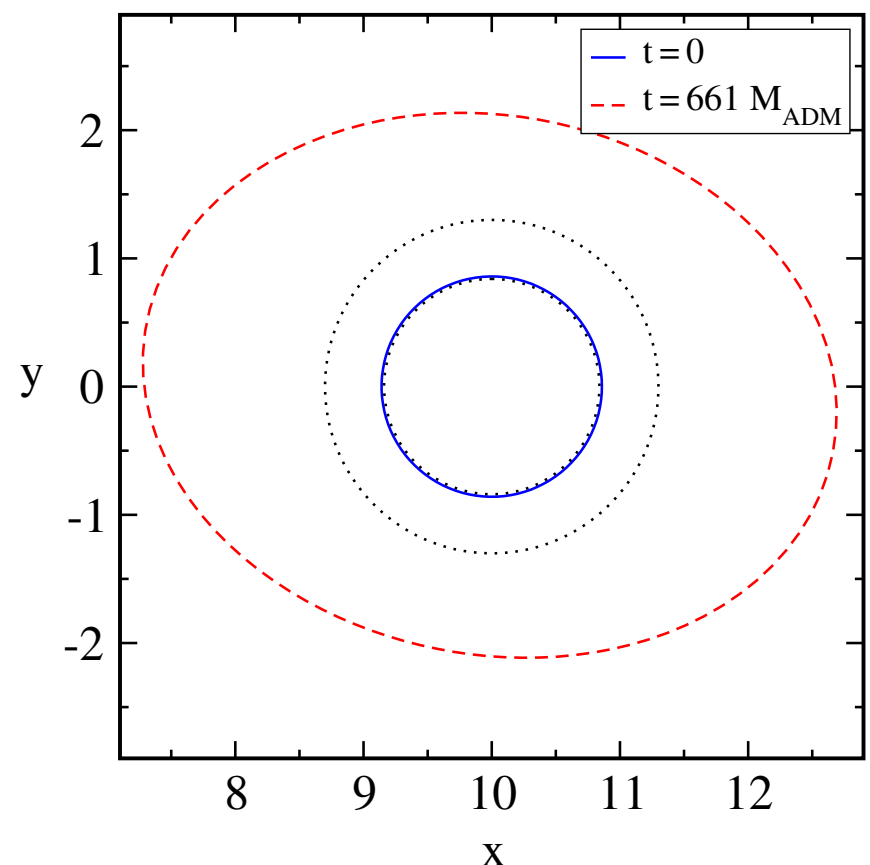

FIG. 13 (color online). Equatorial cross sections, in comoving coordinates, of the apparent horizon of one hole for the evolution of Fig. 10. The horizon is shown both at the beginning and at the end of the evolution. The smaller dotted circle (just inside the initial horizon) shows the excision boundary used for $t \leq$ $554.1 M_{\mathrm{ADM}}$. The outer dotted circle shows the excision boundary used for $t>554.1 M_{\mathrm{ADM}}$.

tion $t=661.4 M_{\mathrm{ADM}}$ (dashed curve). The conformal factor $a(t)$ used to keep the black holes at a fixed coordinate separation also causes the apparent horizons to expand in comoving coordinates. As the binary evolves, the excision boundary (which has a fixed coordinate radius in the moving frame) moves deeper and deeper into the interior of the black hole. At fixed numerical resolution, this leads to rapidly increasing truncation errors that in turn generate constraint violations. The dominant source of constraint violations seen at the end of the evolutions in Fig. 12 comes from the region within the apparent horizons of the two black holes. This problem was partially corrected by moving the location of the excision boundaries at the time $t=$ $554.1 M_{\mathrm{ADM}}$ : for each hole, a portion of the unphysical interior grid was removed (by interpolating the dynamical fields onto a smaller grid), thus moving the excision boundary to the location of the outer dotted curve seen in Fig. 13. This evolution stops in part because truncation errors grow rapidly as the excision boundaries move deeper inside the holes. Another reason this evolution stops can also be seen in Fig. 13: the shape of each apparent horizon has become very distorted by tidal interaction with the companion hole. This distortion requires a significant increase in angular resolution to represent it accurately; so at fixed numerical resolution, truncation errors quickly grow and these cause rapidly growing constraint violations seen near the end of the evolutions in Fig. 12. 


\section{DISCUSSION}

We have shown in Sec. III that the dual-coordinate frame evolution method introduced in Sec. II is capable of solving the rotating-frame instability problem. A more sophisticated application of this method has also allowed us to construct comoving coordinates in Sec. V that track the apparent horizons of a binary black-hole system through a feedback control system. These horizon-tracking coordinates allowed us to evolve a binary black-hole system stably and accurately for about 4.6 orbits. Our expectation is that the great flexibility of the dual-coordinate method will make it useful in many other applications. We imagine, for example, that coordinate maps could be constructed that keep the shape of the apparent horizons spherical, and their locations close to the excision boundary. Such maps should solve the problems that prevent the binary black-hole evolution shown here from proceeding all the way to merger. We also expect that many applications are possible that we are not able to anticipate at this point.

\section{ACKNOWLEDGMENTS}

This work was supported in part by a grant from the Sherman Fairchild Foundation to Caltech and Cornell, by NSF Grants No. PHY-0099568, No. PHY-0244906, and No. PHY-0601459, and NASA Grants No. NAG5-12834, No. NNG05GG52G at Caltech, and by NSF Grants No. PHY-0312072, No. PHY-0354631, and NASA Grant No. NNG05GG51G at Cornell.

\section{APPENDIX}

In this Appendix, we describe some of our efforts to understand and cure the instability that occurs when solving the generalized harmonic system using a single rotating-coordinate frame. We found the instability, illustrated in Fig. 1, to occur even for evolutions of flat spacetime in a rotating frame. Since Minkowski space is simpler than Schwarzschild, much of our analysis was focused on understanding the flat-space case.

At the analytical level, it is easy to see that in Minkowski-space stability in nonrotating inertial coordinates is equivalent to stability in a single rotatingcoordinate frame. The basic argument is as follows. Consider two coordinate systems $x^{\bar{a}}$ and $x^{a}$ related by a uniform rotation, Eqs. (7)-(10). The evolution equations for linear perturbations of the metric $\delta \psi_{a b}$ about a flat Minkowski background may be written in an abstract way as $E_{a b}[\delta \psi]=0$. We assume that both the fields and these evolution equations transform as tensors, i.e., $\delta \psi_{a b}=A_{a}{ }^{\bar{a}} A_{b}{ }^{\bar{b}} \delta \psi_{\bar{a} \bar{b}}$ and $E_{a b}=A_{a}{ }^{\bar{a}} A_{b}{ }^{\bar{b}} E_{\bar{a} \bar{b}}$, where $A_{a} \bar{a} \equiv \partial x^{\bar{a}} / \partial x^{a}$. This implies that if $\delta \psi_{\bar{a} \bar{b}}$ is a solution in the inertial frame then $\delta \psi_{a b}=A_{a}{ }^{\bar{a}} A_{b}{ }^{\bar{b}} \delta \psi_{\bar{a} \bar{b}}$ is a solution in the rotating frame. Suppose that $\delta \psi_{\bar{a} \bar{b}} \sim e^{s \bar{t}} e^{i m \bar{\varphi}}$ is an eigenmode in the inertial frame. Then $\delta \psi_{a b} \sim e^{s^{\prime} t} e^{i m \varphi}$ is a solution in the rotating frame with $s^{\prime}=s-i m \Omega$, so that $\operatorname{Re}\left(s^{\prime}\right)=\operatorname{Re}(s)$. Thus, linear stability in the two frames is equivalent. We have done a complete linear stability analysis in the inertial frame, and the solutions are (of course) stable. Nevertheless, our generalized harmonic evolution code is stable for nearly flat evolutions in nonrotating coordinates, but is extremely unstable for solutions in a rotating frame.

To understand this apparent contradiction, let us now consider the second-order form of the vacuum generalized harmonic evolution equations in more detail:

$$
E_{a b}[\psi]=R_{a b}[\psi]-\nabla_{(a} \mathcal{C}_{b)}[\psi]=0,
$$

where $\psi_{a b}$ is the four-metric, $\nabla_{a}$ its covariant derivative, $R_{a b}$ the Ricci tensor, and $\mathcal{C}_{a}$ is the harmonic constraint defined in Eq. (6). Equation (A1) transforms as a tensor if and only if $\mathcal{C}_{a}$ transforms as a vector. For this to be true, we have to demand a rather special transformation law for the gauge source function $H_{a}$ : from the known transformation of the Christoffel symbols and Eq. (6) we deduce

$$
H_{a}=\tilde{H}_{a}-\psi_{a d} \psi^{b c} \hat{\Gamma}_{b c}^{d},
$$

where $\tilde{H}_{a} \equiv A_{a}{ }^{\bar{a}} H_{\bar{a}}$ and $\hat{\Gamma}_{b c}^{a} \equiv\left(A^{-1}\right)^{a}{ }_{\bar{a}} \partial_{c} A_{b}{ }^{\bar{a}}$. Naively, in order to evolve Schwarzschild spacetime in a rotating frame, say, one would just set $H_{a}$ to be the $-\Gamma_{a}$ of the exact solution evaluated in that frame and freeze it in time. In contrast, Eq. (A2) tells us that $H_{a}$ should depend on the evolved metric $\psi_{a b}$. We find that freezing the quantity $\tilde{H}_{a}$ in the rotating frame and then obtaining $H_{a}$ from Eq. (A2) using the evolved metric $\psi_{a b}$ makes our rotating-frame Minkowski and Schwarzschild evolutions much more (but not completely) stable.

Our numerical code solves a first-order representation of the generalized harmonic evolution equations. To understand the remaining problems, we must look at the firstorder reduction in more detail. The first-order formulation introduces the new dynamical fields

$$
\Pi_{a b} \equiv-t^{c} \partial_{c} \psi_{a b}, \quad \Phi_{k a b} \equiv \partial_{k} \psi_{a b} .
$$

Unlike the metric $\psi_{a b}$, these new fields do not transform as tensors, as can be appreciated from Eqs. (3) and (4). Thus, the simple analytical argument given above for the stability of rotating flat space does not apply. We can modify the standard first-order form of the equations by changing the definitions of these new fields slightly. We replace Eq. (A3) with

$$
\tilde{\Pi}_{a b} \equiv-t^{c} \hat{\nabla}_{c} \psi_{a b}, \quad \tilde{\Phi}_{k a b} \equiv \hat{\nabla}_{k} \psi_{a b},
$$

where $\hat{\nabla}_{a}$ denotes the covariant derivative associated with the connection $\hat{\Gamma}_{b c}^{a}$ introduced in Eq. (A2). By definition, $\hat{\nabla}_{a}$ reduces to $\partial_{a}$ in the inertial frame, but not in the rotating frame. The modified variables in Eq. (A4) by definition transform as tensors under rotations of the coordinates. To make this new first-order formulation truly 
covariant we must also ensure that the evolution equations (and boundary conditions) transform as tensors. To do this, we start with the equations written in the inertial frame (see e.g., Eqs. [35]-[37] of Ref. [5]) and formally replace all partial derivatives $\partial_{a}$ with the covariant derivatives $\hat{\nabla}_{a}$. The rotating frame solutions of these modified evolution equations should then have the same stability properties as the solutions obtained in the inertial frame. We have used this system to evolve both Minkowski and Schwarzschild spacetimes, and found the numerical solutions to be greatly improved over the unmodified system. But the results are still not quite stable.

The next problem that we discovered turned out to be numerical: we needed to modify the standard tensorspherical-harmonic filtering algorithm described, for example, in Sec. III. The transformation that relates the rotating frame components of tensors with nonrotating frame components mixes together the time components with some spatial components (e.g., $\psi_{t t}$ depends on $\psi_{\bar{t} \bar{t}}$, $\psi_{\bar{t} \bar{\varphi}}$, and $\left.\psi_{\bar{\varphi} \bar{\varphi}}\right)$. This causes scalar, vector, and second-rank tensor spherical harmonics to be mixed together in a non- linear way. To disentangle these parts, it is necessary first to transform the time components of these fields to the inertial frame, filter them there, and then transform them back to the rotating frame. In this way, we remove the mixing of the different tensor spherical harmonics that occurs in a rotating frame. Using this filtering method (which turns out to be equivalent to the filtering used for our dualcoordinate evolutions described in Sec. III) produces Minkowski-space evolutions that appear to be completely stable, while evolutions of Schwarzschild though improved are still not quite stable.

The combination of the modifications described above significantly improves the stability of our evolutions of flat space and single black-hole spacetimes in rotating coordinates. However, these methods did not produce robustly stable evolutions over the entire range of domain sizes and rotating frame angular velocities needed for binary blackhole simulations. We stopped pursuing this approach after the dual-frame method was found to be so stable, and so flexible that it allowed us to solve our moving frame excision problem as well.
[1] F. B. Estabrook, R. S. Robinson, and H.D. Wahlquist, Classical Quantum Gravity 14, 1237 (1997).

[2] H. Friedrich and G. Nagy, Commun. Math. Phys. 201, 619 (1999).

[3] L. T. Buchman and J.M. Bardeen, Phys. Rev. D 67, 084017 (2003).

[4] J. R. Wilson, in Sources of Gravitational Radiation, edited by L. Smarr (Cambridge University Press, Cambridge, England, 1979), pp. 423-445.

[5] L. Lindblom, M. A. Scheel, L. E. Kidder, R. Owen, and O. Rinne, Classical Quantum Gravity 23, S447 (2006).

[6] F. Pretorius, Phys. Rev. Lett. 95, 121101 (2005).

[7] G. B. Cook et al., Phys. Rev. Lett. 80, 2512 (1998).

[8] S. Brandt et al., Phys. Rev. Lett. 85, 5496 (2000).

[9] M. Alcubierre and B. Brügmann, Phys. Rev. D 63, 104006 (2001).

[10] D. Shoemaker, K. Smith, U. Sperhake, P. Laguna, E. Schnetter, and D. Fiske, Classical Quantum Gravity 20, 3729 (2003).

[11] M. Campanelli, C. O. Lousto, P. Marronetti, and Y. Zlochower, Phys. Rev. Lett. 96, 111101 (2006).

[12] J. G. Baker, J. Centrella, D.-I. Choi, M. Koppitz, and J. van Meter, Phys. Rev. Lett. 96, 111102 (2006).

[13] M. Campanelli, C. O. Lousto, and Y. Zlochower, Phys. Rev. D 73, 061501(R) (2006).

[14] J. G. Baker, J. Centrella, D.-I. Choi, M. Koppitz, and J. van Meter, Phys. Rev. D 73, 104002 (2006).

[15] M. Campanelli, C. O. Lousto, and Y. Zlochower, Phys.
Rev. D 74, 041501 (2006).

[16] J.R. van Meter, J. G. Baker, M. Koppitz, and D.-I. Choi, Phys. Rev. D 73, 124011 (2006).

[17] R. Gomez, L. Lehner, R. L. Marsa, and J. Winicour, Phys. Rev. D 57, 4778 (1998).

[18] L.E. Kidder, M. A. Scheel, and S.A. Teukolsky, Phys. Rev. D 64, 064017 (2001).

[19] L. E. Kidder, L. Lindblom, M. A. Scheel, L. T. Buchman, and H. P. Pfeiffer, Phys. Rev. D 71, 064020 (2005).

[20] M. Boyle, L. Lindblom, H. P. Pfeiffer, M. A. Scheel, and L. E. Kidder, gr-qc/0609047 [Phys. Rev. D (to be published)].

[21] M. Holst, L. Lindblom, R. Owen, H. P. Pfeiffer, M. A. Scheel, and L. E. Kidder, Phys. Rev. D 70, 084017 (2004).

[22] G. B. Cook and H.P. Pfeiffer, Phys. Rev. D 70, 104016 (2004).

[23] http://black-holes.org/researchers3.html.

[24] T. W. Baumgarte, G. B. Cook, M. A. Scheel, S. L. Shapiro, and S. A. Teukolsky, Phys. Rev. D 54, 4849 (1996).

[25] H. P. Pfeiffer, S. A. Teukolsky, and G. B. Cook, Phys. Rev. D 62, 104018 (2000).

[26] H. P. Pfeiffer, G. B. Cook, and S. A. Teukolsky, Phys. Rev. D 66, 024047 (2002).

[27] E. D. Sontag, Mathematical Control Theory: Deterministic Finite Dimensional Systems, in Textbooks in Applied Mathematics, No. 6 (Springer, New York, 1998), 2nd ed. 